\title{
Maxillary Sinus Squamous Cell Carcinoma: A Clinical Study
}

\author{
Nobusuke Hohchi ${ }^{1}$ Tetsuro Wakasugi ${ }^{1}$ Fumiko Takenaga ${ }^{1}$ Shoko Takeuchi ${ }^{1}$ Jun-ichi Ohkubo ${ }^{1}$ \\ Hiroki Koizumi $^{1}$ Toyoaki Ohbuchi ${ }^{1}$ Hideaki Suzuki $^{1}$ \\ 1 Department of Otorhinolaryngology and Head and Neck Surgery, \\ University of Occupational and Environmental Health School of \\ Medicine, Yahatanishi-ku, Kitakyushu, Fukuoka, Japan \\ Int J Pract Otolaryngol 2018;1:e10-e15.

\begin{abstract}
Address for correspondence Nobusuke Hohchi, MD, Department of Otorhinolaryngology and Head and Neck Surgery, University of Occupational and Environmental Health School of Medicine, 1-1 Iseigaoka, Yahatanishi-ku, Kitakyushu, Fukuoka, 807-8555, Japan
\end{abstract} \\ (e-mail: houchi@med.uoeh-u.ac.jp).
}

\begin{abstract}
Keywords

- maxillary sinus squamous cell carcinoma

- treatment outcome

- survival rate

- superselective intra-arterial chemoradiotherapy

We retrospectively analyzed the clinical characteristics and treatment outcomes of patients with maxillary sinus squamous cell carcinoma. The patients were 22 newly diagnosed cases of maxillary sinus squamous cell carcinoma who were treated at our institute between 2005 and 2016. There were 19 males and 3 females, ranging in age from 45 to 83 years (average age: 64.2 years). Our treatment protocol was as follows: All patients received chemoradiotherapy with weekly superselective intra-arterial administration of cisplatin (radiotherapy and concomitant intra-arterial cisplatin [RADPLAT]; 100-120 mg/body). Good responders at 30 to 40 Gy received additional radiotherapy (RADPLAT, ordinary chemoradiotherapy, or radiotherapy alone) until the total dose reached $72 \mathrm{~Gy}$, whereas the other patients underwent radical surgery (partial maxillectomy, total maxillectomy, or extended total maxillectomy). The latter patients were given postoperative (chemo)radiotherapy up to a total dose of $72 \mathrm{~Gy}$, when histopathological examination revealed insufficient resection or a positive surgical margin. The overall 5 -year crude and disease-specific survival rates determined by the Kaplan-Meier method were 47.8 and $56.9 \%$, respectively. The crude survival rate tended to be higher in T3 patients than in T4 patients ( 80.0 vs. $29.9 \%$, $p=0.077$ ). Seven patients died of the primary disease. The cause of death was distant metastasis in four patients, recurrence of the primary lesion in two patients, and cervical lymph node metastasis in one patient. These results suggest the importance of early detection and early treatment, and of the development of more effective treatment for advanced maxillary cancers, to further improve the survival rate.
\end{abstract}

\section{Introduction}

The paranasal cavity is anatomically situated in proximity to the esthetically important organs, such as visual organs and the face. Malignant tumors that develop in the paranasal cavity are often difficult to treat; it is also challenging to ensure an adequate safety margin while performing surgery. Other factors complicating treatment include the facts that (1) subjective symptoms are negligible in the early stage, (2) the disease highly advances in several patients even before a diagnosis is made, and (3) various histopathological types are presented. Generally, multi- modal treatment combines surgery, radiotherapy, and (intraarterial) chemotherapy. Moreover, newly developed chemotherapies, including molecular-targeting drugs and advancements in radiotherapy such as proton beam, baryon beam, and intensity-modulated radiotherapy (IMRT), have recently been reported. However, compared with oral, pharyngeal, and laryngeal cancers, few patients present with paranasal cavity cancer, and it is difficult to say that the treatment method is established. Here, we examined and reported the clinical characteristics and treatment outcomes of maxillary sinus squamous cell carcinoma observed in inpatients at our hospital. received

November 13, 2017

accepted

May 22, 2018
DOI https://doi.org/

10.1055/s-0038-1669404. eISSN 2569-1783. (c) 2018 Georg Thieme Verlag KG
Stuttgart · New York

License terms

(c) $(1) \$$ 


\section{Materials and Methods}

The study included 22 (males, 19; females, 3; age, 4583 years [mean age, 64.2 years]) patients diagnosed with and treated for maxillary sinus squamous cell carcinoma at the Department of Otorhinolaryngology and Head and Neck Surgery, University of Occupational and Environmental Health School of Medicine during the 11-year period between January 2005 and 2016. Patients with recurrence and a treatment history at any other institution were excluded. The mean observation period was 34.3 months.

Disease stage was determined based on the Union for International Cancer Control tumor node metastasis (UICC TNM) classification (7th ed.), and treatment outcomes were evaluated according to overall and disease-specific survival. The Kaplan-Meier method was used to analyze the survival rate, and significant differences in survival rate were verified using log-rank test. $p<0.05$ was considered statistically significant, and $p<0.1$ was considered a trend.

The basic policy for treating primary lesions followed by our department during the study period is presented in - Fig. $\mathbf{1}$. First, preoperative superselective intra-arterial chemotherapy (radiotherapy and concomitant intra-arterial cisplatin [RADPLAT]) was administered. Intra-arterial infusion was administered in accordance with the Seldinger method, whereby a catheter was inserted via the right femoral artery and tumorfeeding vessels, such as the maxillary and facial arteries, were identified. Thereafter, cisplatin (CDDP) was administered via a microtube inserted into these blood vessels at a dose of 100 to $120 \mathrm{mg} /$ body surface area $\left(\mathrm{m}^{2}\right)$ per week, total two to four times (mean doses, three). Radiation was given via the simple fractionated irradiation technique $(2.0 \mathrm{~Gy} /$ fraction, 1 fraction/ day), and when doses of 30 to $40 \mathrm{~Gy}$ were received, the primary lesion was evaluated according to the response evaluation criteria in solid tumors. In patients exhibiting good response in this interim evaluation (complete response [CR]:
Table 1 Number of patients according to each TN stage

\begin{tabular}{|l|l|l|l|l|l|}
\hline T/N & N0 & N1 & N2 & N3 & Total \\
\hline T1 & 0 & 0 & 0 & 0 & 0 \\
\hline T2 & 0 & 0 & 0 & 0 & 0 \\
\hline T3 & 4 & 0 & 2 & 0 & 6 \\
\hline T4 & $11\left(1^{\mathrm{a}}\right)$ & 3 & 2 & 0 & 16 \\
\hline Total & 15 & 3 & 4 & 0 & 22 \\
\hline
\end{tabular}

${ }^{a}$ Patients with distal metastasis are shown in parentheses.

disappearance or good partial response [good PR]: cicatrization) and in those unable to or refusing to undergo surgery, treatment was continued by either RADPLAT, chemoradiotherapy (CRT), or radiotherapy alone (RT) until 72 Gy. Among patients exhibiting other responses (PR:tumor reduction/stable disease [SD]:unchanged/progressive disease [PD]: exacerbation) and those who were able to undergo surgery, radical surgery by partial maxillectomy, total maxillectomy, or extended maxillectomy ( \pm extensive skull base tumorectomy and reconstruction) was selected in accordance with the extent of progression of the primary lesion. Following surgery, based on the histopathology of the surgical specimen, CRT or RT was administered until a total dose of $72 \mathrm{~Gy}$ was reached.

\section{Results}

\section{Patients' Clinical Characteristics and Treatment Outcomes}

\section{Clinical Staging}

The number of patients chosen for each $\mathrm{T}$ and $\mathrm{N}$ classification is listed in - Table 1. No patients exhibited T1 or T2 disease, 6 (21.4\%) exhibited T3 disease, and 16 (72.7\%) exhibited T4 disease. The staging classification was stage III in 4 patients

\section{Non-surgery: 5 patients \\ CR/good PR: 2 patients \\ Surgery refused: 1 patients \\ Advanced progression: 2 patients}

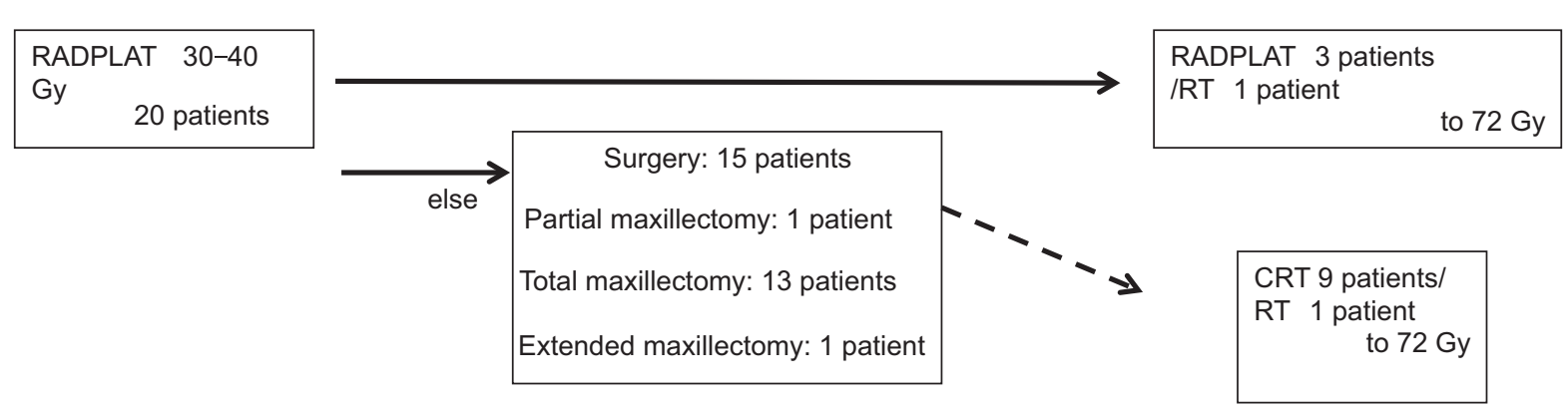

Fig. 1 Basic treatment policy for primary lesions of maxillary sinus squamous cell carcinoma followed at our department. RADPLAT, radiotherapy and concomitant intra-arterial cisplatin; RT, radiotherapy alone; CRT, chemoradiotherapy; Surgery, partial maxillectomy, total maxillectomy, and extended maxillectomy ( \pm extensive skull base tumorectomy and reconstruction); $C R$, complete response and disappearance; good PR, good partial response and cicatrization. 
and stage IV in 18 (IVA, 15 patients; IVB, 2 patients; and IVC, 1 patient), thus indicating that all patients were in the advanced disease stage.

\section{First-Line Treatment}

As the first-line treatment, RADPLAT was performed in 20 patients (90.9\%), CRT in one patient who received concurrent treatment for hypopharyngeal cancer, and partial maxillectomy in one patient considering the general condition.

Among the 20 patients who received RADPLAT, radical surgery was performed in 15 (T3, 4 patients; T4, 11 patients; $70 \%)$. Surgery included total maxillectomy in 13 patients (65.0\%), partial maxillectomy in 1 (5.0\%), and extended total maxillectomy + extensive skull base tumorectomy and reconstruction in 1 (5.0\%). Nonsurgery patients included two patients with $\mathrm{CR} /$ good PR on the interim evaluation, one (5.0\%) who refused surgery, and two (10.0\%) with highly advanced disease who were judged unable to undergo surgery (one patient with clivus invasion and another with invasion into the orbital apex).

\section{Treatment Outcomes}

Overall survival and disease-specific survival curves of maxillary sinus squamous cell carcinoma are presented in - Fig. 2. For all patients, the 5-year overall survival rate was $47.8 \%$, whereas the 5 -year disease-specific survival rate was $56.9 \%$. The overall survival and disease-specific survival curves according to $\mathrm{T}$ classification are presented in - Figs. 3 and $\mathbf{4}$. The 5-year overall survival rate was $80.0 \%$ in T3 patients; however, this rate was significantly lower in T4 patients (29.9\%; $p=0.077)$. The 5 -year disease-specific survival rate was $80.0 \%$ in $\mathrm{T} 3$ patients and was lower at $42.3 \%$ in T4 patients; however, the difference observed was not significant ( $p=0.133$ ).

In the 20 patients who received RADPLAT, the 5-year overall survival rate was $47.9 \%$, whereas the 5 -year disease- specific survival rate was $59.8 \%$. Among the 15 patients who received RADPLAT and radical surgery, the 5-year overall survival rate was $59.4 \%$, whereas the 5 -year disease-specific survival rate was $71.8 \%$.

During initial examination, seven patients had $\mathrm{N}+$ disease. In the $\mathrm{N}$ - group, the 5-year overall survival rate was $53.2 \%$ and the 5 -year disease-specific survival rate was $63.3 \%$. In the $\mathrm{N}+$ group, the 5 -year overall survival rate and diseasespecific survival rate were lower at 33.3\%; however, the difference observed was not significant (overall survival rate, $p=0.472$; disease-specific survival rate, $p=0.397$ ).

\section{Outcomes}

Of the 22 patients, 9 patients died ( 7 cancer-specific deaths and 2 due to other causes). The cancer-specific deaths included T-related death in two patients, N-related death in one, and M-related death in four.

\section{Discussion}

The incidence of primary malignancy of the nasal and paranasal cavity is relatively rare, affecting 0.5 to 1.0 per 100,000 individuals; further, primary malignancy of the nasal and paranasal cavity is said to account for approximately $0.5 \%$ of all malignancies and $3 \%$ of head and neck cancers. ${ }^{1}$ The incidence of maxillary sinus carcinoma is also low. Furthermore, while there is no clearly established evidence owing to very few prospective clinical treatment trials conducted worldwide, surgery-based treatment is recognized as the standard treatment in Europe and the United States. Surgery (total maxillectomy and extended total maxillectomy) is considered the standard treatment when resection is possible, and many patients receive postoperative radiotherapy. For overseas, patients in whom resection and surgery are not possible, CRT is generally administered. ${ }^{2}$

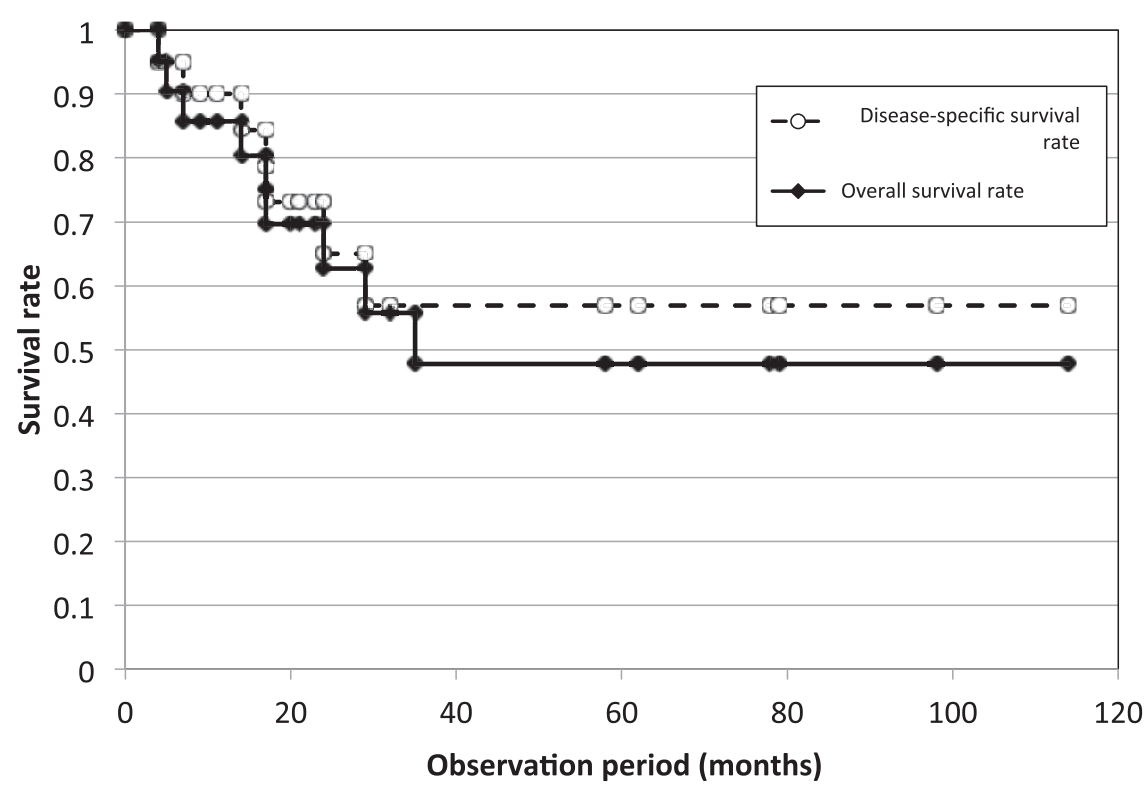

Fig. 2 Overall survival and disease-specific survival for maxillary sinus squamous cell carcinoma. The 5 -year overall survival was $47.8 \%$, whereas the 5-year disease-specific survival was 56.9\%. 


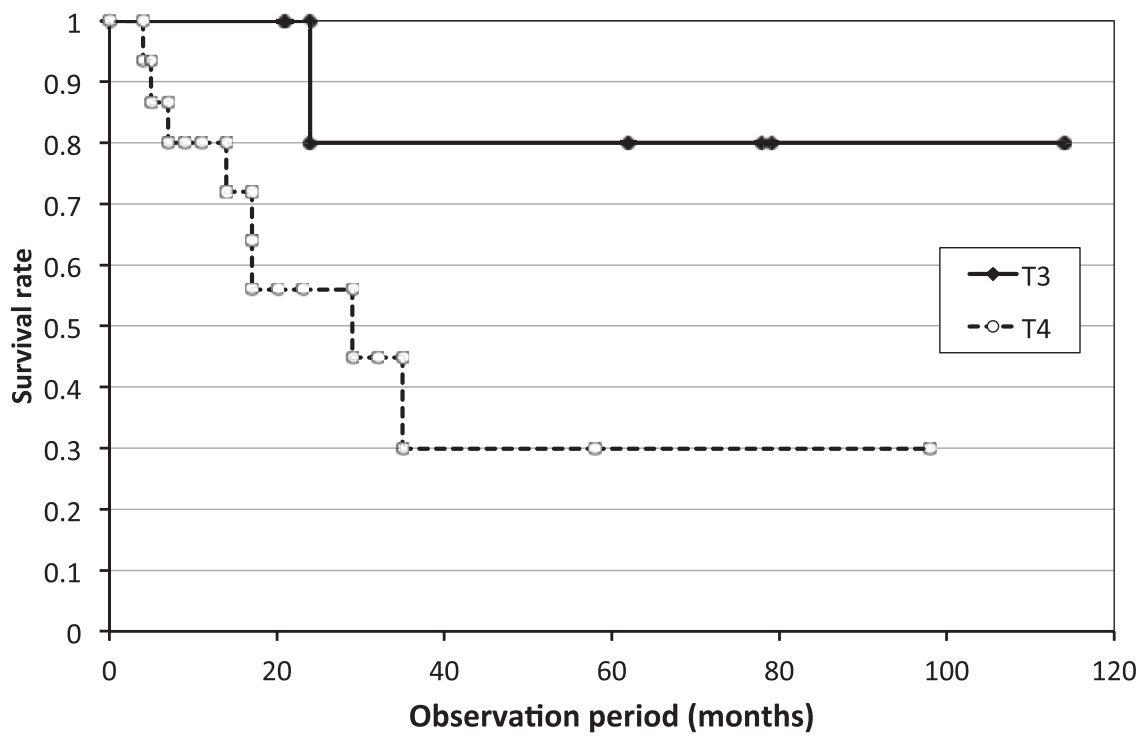

Fig. 3 Overall survival rate according to T-staging of maxillary sinus squamous cell carcinoma. The 5 -year overall survival was $80.0 \%$ in T3 patients and $29.9 \%$ in T4 patients $(p=0.077)$.

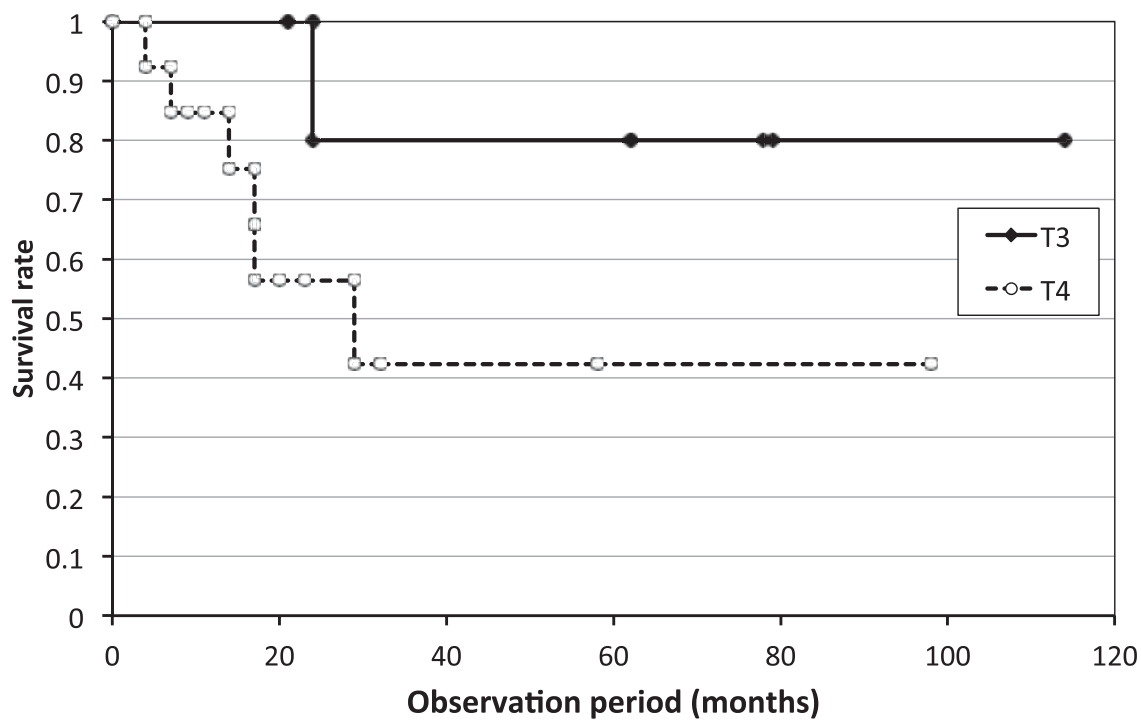

Fig. 4 Disease-specific survival rate according to T-staging of maxillary sinus squamous cell carcinoma. The 5 -year overall survival was $80.0 \%$ in T3 patients and $42.3 \%$ in T4 patients $(p=0.133)$.

At our department, the 5-year overall survival rate was $47.8 \%$ and the disease-specific survival rate was $56.9 \%$ for all patients with maxillary sinus squamous cell carcinoma. Several Japanese studies reported the 5 -year survival rate to be 44.0 to $57.0 \%,{ }^{3-5}$ and our results corroborated with those reported in these studies.

The histopathological types of nasal and paranasal cavity malignancies are more varied compared with those of laryngeal and pharyngeal malignancies; however, most instances are of squamous cell carcinoma. This is attributable to the fact that the mucosa of the nasal and paranasal cavities consists of ciliated pseudostratified columnar epithelium, which develops into squamous epithelium where cancer develops. ${ }^{6}$ Squamous cell carcinoma responds relatively well to CRT; therefore, multimodal treatment is administered.
Regarding the degree of progression of the primary lesion in maxillary sinus carcinoma, several studies reported to date indicate that $\mathrm{T} 3$ disease or greater is common. ${ }^{7-9}$ In our reports also, all patients had $\mathrm{T} 3$ disease or greater. T-staging has the greatest impact as a prognosticator of maxillary sinus carcinoma, ${ }^{10}$ and reportedly, prognosis is significantly worse in patients with T4 disease than with $\mathrm{T} 3$ or lesser. ${ }^{4-6,8,9}$ In the present study, the 5-year overall survival rate in $\mathrm{T} 3$ patients was $80.0 \%$; however, the rate reduced to $29.9 \%$ in patients with T4 disease. Maxillary sinus carcinoma with the tumor limited to within the sinus (T1/T2) presents no symptoms or symptoms that barely differ from those of sinusitis; further, it is difficult to detect T1/T2 early-stage maxillary sinus carcinoma. By the time specific symptoms, such as foul-smelling nasal discharge, buccal pain, dental 
pain, and buccal redness and swelling, are observed, many patients would have had already developed T3/T4 disease. It is common for patients to observe and ignore symptoms such as nasal congestion and nasal flow; further, when buccal pain and swelling appears, many patients consult a dentist thinking it is a tooth-related problem. From a physician's perspective, general nasal symptoms should not be neglected; particularly, when the symptoms are unilateral, image examination such as computed tomography should be proactively performed. To detect maxillary sinus carcinoma well in advance, we believe it is important to educate the society in general and provide information to not only departments of otorhinolaryngology but also to medical departments of adjacent areas, such as the department of dentistry and ophthalmology.

The incidence of $\mathrm{N}+$ disease at the initial examination is reported to be 6 to 22\% in maxillary sinus carcinoma, and our result of seven patients (31.8\%) is somewhat higher than the usual. In the process of evaluating prognosis according to the presence or absence of cervical lymph node metastasis, Maeda et $\mathrm{al}^{8}$ reported that the 5 -year disease-specific survival rate is $63 \%$ in $\mathrm{N}$ - patients and $18 \%$ in $\mathrm{N}+$ patients, thereby indicating poorer prognosis in $\mathrm{N}+$ patients. By contrast, Asakura et $\mathrm{al}^{11}$ reported that the survival rate does not depend on cervical lymph node metastasis and that control of the primary lesion is more important. Our results indicated that the 5-year overall survival rate was lower in $\mathrm{N}+$ patients; however, this difference was not statistically significant. In the future, an ongoing follow-up survey should be conducted with a larger number of samples.

The basic treatment method for maxillary sinus squamous cell carcinoma at our department, as shown in - Fig. 1, is preoperative RADPLAT combined with surgery. A comparative study conducted in Holland ${ }^{12}$ revealed that RADPLAT is not superior to CRT; therefore, administration of RADPLAT has recently ceased in the West. ${ }^{13}$ On the contrary, RADPLAT is generally used to treat maxillary sinus carcinoma in Japan. Similar to that performed at our institution, Koike et al administered preoperative RADPLAT using the Seldinger method combined with surgery and reported a 5-year disease-specific survival rate of $100 \%$ in T3 patients and $84.7 \%$ in T4 patients, indicating good outcomes. ${ }^{14}$ Shiga et al administered RADPLAT via superselective intra-arterial chemotherapy for maxillary sinus squamous cell carcinoma and reported no difference in survival in the group with surgery compared with in the group without surgery and radiation therapy up to the curative dose; further, they reported good outcomes in both groups and improved prognosis in stage IV patients. ${ }^{7,15}$ Based on these reports, we can adequately infer that RADPLAT has a certain effectiveness.

Among the patients at our department, long-term survival was achieved in two patients with ongoing RADPLAT without surgery. In the interim evaluation, good PR was observed in one patient with T3 disease and one with T4b disease who was unable to undergo surgery. Currently, both these patients are alive and recurrence free. While it is hard to draw a conclusion owing to the small sample size, we believe that continuing using RADPLAT for patients who responded well to RADPLAT based on the interim evaluation might be sufficient to preserve the maxilla and achieve longterm survival. Particularly, it should be noted that CR was achieved in a patient with T4b disease, which corroborates with the finding reported by Shiga, ${ }^{7}$ thus indicating that RADPLAT monotherapy should be considered in the treatment of advanced maxillary sinus carcinoma.

The dose/CDDP infusion using the original method reported by Robbins et al is $150 \mathrm{mg} / \mathrm{m} ;{ }^{2,16}$ however, CDDP is often administered at a dose of $100 \mathrm{mg} / \mathrm{m}^{2}$ in Japan. Yoshizaki et al compared the outcomes achieved with CDDP doses of 100 and $150 \mathrm{mg} /$ body surface area and observed that the outcomes were better with the latter dose; furthermore, they discussed the need for high-dose CDDP administration because maxillary sinus carcinoma has a greater tumor volume compared with that of laryngeal or hypopharyngeal cancer. ${ }^{17}$ Moreover, the Japan Clinical Oncology Group conducted a multicenter, prospective clinical trial of maxillary sinus carcinoma in Japan and reported that RADPLAT was effective for localized advanced maxillary sinus squamous cell carcinoma and that cisplatin administered at a dose of $100 \mathrm{mg} / \mathrm{m}^{2}$ for seven cycles is safe and stable. ${ }^{18}$ To date, at our department, we have mainly administered CDDP arterial infusion at a dose of $100 \mathrm{mg} /$ body surface area; however, for localized, highly advanced patients, we believe that increasing the CDDP dosage will ease further examination. On the contrary, a certain incidence of severe adverse events of RADPLAT including cerebrovascular accidents, central nervous system disorder, and respiratory stenosis, as well as late adverse events including osteonecrosis, brain necrosis, and severe visual impairment, which are irreversible adverse events that decrease quality of life, has been reported. ${ }^{19}$ Among all the patients at our department, one patient had temporary visual impairment and another had facial nerve paralysis; however, there was no occurrence of the abovementioned severe adverse events. Both these patients recovered; we believe that in the future, the possibility of the onset of late adverse events should be recognized and careful follow-up observation should continue over a long period.

The outcomes observed via the current treatment, which combined RADPLAT with surgery, used at our department were generally good for maxillary sinus carcinoma up to the T3 disease. However, in highly advanced patients (T4), the disease is often difficult to control, and more treatment methods need to be developed. Upon examining fatal cases, the most common cause of death was found to be M-related, followed by T-related deaths. As presented in - Table 1, patients with distal metastasis (+) at the time of initial examination were in the T4 stage; in advanced patients, appropriate intervention via systemic chemotherapy might be needed from the time of initial treatment. Therefore, a more effective chemotherapy regimen needs to be established in the future. Noronha et $\mathrm{al}^{20}$ achieved certain outcomes by treating patients with localized advanced cancer with systemic chemotherapy followed by surgery and radiotherapy. Furthermore, the first-line treatment is important for improving the primary lesion control rate. Because the center of the face is adjacent to the orbit and the cranium, and particularly in advanced maxillary sinus carcinoma, performing surgery while ensuring an adequate safety margin is often 
functionally and esthetically difficult, which we believe leads to T-related death. To improve the localized control rate, we believe it might be useful to examine the use of surgeryassisting tools, such as endoscopy and navigation systems, which have made remarkable advancements recently, as well as the concomitant use of advanced radiotherapies, such as IMRT and particle beam radiotherapy.

\section{Conclusion}

We examined 22 patients with maxillary sinus squamous cell carcinoma who underwent first-line treatment at our department. The 5-year overall survival and disease-specific survival rates were 47.8 and $56.9 \%$, respectively. The survival rate was good up to the T3 disease stage and then declined in the T4 disease stage. In fatal cases, the most common cause of death was M-related, followed by Trelated death. We believe that improving the survival rate in the future involves early detection and treatment, establishing improved treatment methods for advanced cancer, and, in particular, controlling the metastatic lesions and the primary lesion.

\section{Conflict of Interest \\ None declared.}

Note

This study was approved by the Ethics Committee of the University of Occupational and Environmental Health.

\section{References}

1 Dulguerov P, Jacobsen MS, Allal AS, Lehmann W, Calcaterra T. Nasal and paranasal sinus carcinoma: are we making progress? A series of 220 patients and a systematic review. Cancer 2001;92 (12):3012-3029

2 National Comprehensive Cancer Network. NCCN clinical practice guidelines in oncology (NCCN Guidelines). Central Nervous System Cancers Version 2. 2011;2:19-21. Available at: http:// oralcancerfoundation.org/treatment/pdf/head-and-neck.pdf. Accessed September 1, 2018

3 Tachida K, Yoshida F, Saijo S, et al. A clinical study of maxillary sinus malignancy. Japanese J Head Neck Cancer 2004;30:8-12

4 Yamashita T, Fujii M, Ishiguro R, et al. [Statistical analysis of maxillary sinus squamous cell carcinoma]. Nippon Jibiinkoka Gakkai Kaiho 2002;105(06):732-740

5 Toshimura T, Ohara H, Ashizawa K, et al. Clinical assessment of paranasal malignant tumor. Jibi Inkoka Tembo 2013;56:49-53
6 Ogawa T. [A clinico-pathological study of adenocarcinomas of the nasal cavity and paranasal sinuses]. Nippon Jibiinkoka Gakkai Kaiho 1989;92(03):317-333

7 Shiga K. What is the treatment selection for patients with T3-4 maxillary sinus squamous cell carcinoma? From the perspective of super-selective arterial infusion. JOHNS 2009;25:1472-1475

8 Maeda A, Chijiwa H, Sakamoto K, Miyajima Y, Umeno H, Nakashima T. [Clinical study of cervical lymph node metastasis in maxillary cancer patients]. Nippon Jibiinkoka Gakkai Kaiho 2008; 111(06):486-489

9 Rikimaru F, Matsuo M, Higaki Y, et al. The treatment of maxillary sinus squamous cell carcinoma at our department. J Japan Soc Head Neck Surg 2011;21:145-149

10 Harbo G, Grau C, Bundgaard T, et al. Cancer of the nasal cavity and paranasal sinuses. A clinico-pathological study of 277 patients. Acta Oncol 1997;36(01):45-50

11 Asakura K, Himi T, Harabuchi Y, et al. A clinical study of maxillary carcinoma. Pract Otorhinolaryngol (Basel) 1995;84:87-95

12 Rasch CR, Hauptmann M, Schornagel J, et al. Intra-arterial versus intravenous chemoradiation for advanced head and neck cancer: results of a randomized phase 3 trial. Cancer 2010;116(09): 2159-2165

13 Homma A. Superselective high-dose cisplatin arterial infusion with concomitant radiotherapy. Japanese J Head Neck Cancer 2012;22:29-31

14 Koike S, Nasu T, Ishida A, et al. Long-term outcomes and late complications of superselective intra-arterial chemotherapy for maxillary squamous cell carcinoma. Japanese J Head Neck Cancer 2010;36:483-487

15 Shiga K, Yokoyama J, Hashimoto S, et al. Combined therapy after superselective arterial cisplatin infusion to treat maxillary squamous cell carcinoma. Otolaryngol Head Neck Surg 2007;136(06): 1003-1009

16 Robbins KT, Storniolo AM, Kerber C, Seagren S, Berson A, Howell SB. Rapid superselective high-dose cisplatin infusion for advanced head and neck malignancies. Head Neck 1992;14(05):364-371

17 Yoshizaki T, Tsukatani T, Wakisaka N, et al. Rapid intra-arterial chemoradiotherapy for the treatment of maxillary sinus cancer: the relationship with CDDP dose. Japanese J Head Neck Cancer 2007;33:434-438

18 Homma A, Onimaru R, Matsuura K, et al. Dose-finding and efficacy confirmation trial of the superselective intra-arterial infusion of cisplatin and concomitant radiotherapy for locally advanced maxillary sinus cancer (Japan Clinical Oncology Group 1212): Dose-finding phase. Head Neck 2018;40(03): 475-484

19 Takahashi G, Mochizuki D, Sugiyama K, et al. A single institution, retrospective study of superselective intra-arterial cisplatin and concomitant radiation for maxillary sinus squamous cell carcinomas [in Japanese]. Pract Otorhinolaryngol 2013;136:5-11

20 Noronha V, Patil VM, Joshi A, et al. Induction chemotherapy in technically unresectable locally advanced carcinoma of maxillary sinus. Chemother Res Pract 2014;2014:487872 